\title{
TCAD advanced radiation damage modeling in silicon detectors
}

\author{
Arianna Morozzi ${ }^{1}$ \\ INFN Perugia \\ Via Pascoli 1, Perugia, Italy \\ E-mail: arianna.morozzi@pg.infn.it
}

\section{Francesco Moscatelli}

CNR-IOM and INFN Perugia

Via Pascoli 1, Perugia, Italy

E-mail: moscatelliliom.cnr.it

\section{Daniele Passeri}

University of Perugia

Via G. Duranti 93, Perugia, Italy

INFN Perugia

Via Pascoli 1, Perugia, Italy

E-mail: daniele.passeri@unipg.it

\section{Gian Mario Bilei}

INFN Perugia

Via Pascoli 1, Perugia, Italy

E-mail: gian.mario.bilei@cern.ch

In this work we present a methodology to develop a surface and bulk TCAD radiation damage effects model which enables a predictive insight into the electrical behavior of novel solid-state detectors up to the particle fluences expected at the end of HL-LHC. To better understand, in a comprehensive framework, the complex and articulated phenomena related to the radiation damage mechanisms several TCAD simulations have been carried out and compared with measurements performed on test structures and sensors. Surface radiation damage effects have been deeply investigated on both p-type and n-type substrate test structures exposed to X-ray irradiation at doses in the range $0.05-100 \mathrm{Mrad}\left(\mathrm{SiO}_{2}\right)$. The complete bulk and surface radiation damage model findings have been then compared with available measurements in terms of charge collection efficiency up to $2.0 \times 10^{16} 1 \mathrm{MeV}$ equivalent $\mathrm{n}_{\mathrm{eq}} / \mathrm{cm}^{2}$.

The 28th International Workshop on Vertex Detectors - Vertex2019

13-18 October, 2019

Lopud, Croatia

\section{${ }^{1}$ Speaker}




\section{Introduction}

The High-Luminosity (HL) era at the Large Hadron Collider (LHC) opens the way to a next generation of tracking detectors which have to withstand extremely high fluences. The luminosity is expected to reach, in the innermost regions of the collider, fluences of the order of $2.2 \times 10^{16} 1$ $\mathrm{MeV} \mathrm{n} \mathrm{n}_{\mathrm{eq}} / \mathrm{cm}^{2}$ and total ionizing doses (TID) up to $1 \mathrm{Grad}$ integrated over 10 years of data taking. Within this scenario, radiation hardness is clearly a critical design constraint for the current and for the next generation of detectors for the inner and outer tracker to cope with the unprecedented increasing radiation levels. To this purpose, the analysis and the comprehension of the complex physical phenomena related to the radiation damage effects in silicon sensors is of utmost importance and device-level analyses represent suitable tools to push forward this comprehension.

Modern Technology CAD (Computer-Aided Design) tools, such as Sentaurus TCAD of Synopsys represent the state-of-the-art numerical approach to predict the electrical behavior of solid-state devices in different operating conditions. Indeed, these tools offer a wide variety of approaches for the design and simulation of semiconductor devices, by suitably balancing physical accuracy and comprehensiveness, application versatility and computational demand. Within this framework bulk and surface radiation damage effects have been modelled by means of the introduction of physically meaningful deep-level radiation-induced traps whose parameterization is based on experimental evidences. By following a physically grounded approach, the proposed methodology aims at keeping the number of fitting parameters as low as possible, thus increasing the model robustness and generality (e.g. in terms of technology flavours, temperature and bias conditions) without overcomplicating the numerical resolution.

The aim of this work is to illustrate how to cope with the complexity of devising a reliable, as well as suitable for provisional analysis model, accounting in a comprehensive way the bulk and surface radiation damage effects. The investigation, at atomistic level, of the phenomenology of the radiation damage mechanisms lies outside the scope of this work which is mainly devoted to the comprehension and prediction of the electrical behavior (namely, at a device level), of the effects related to radiation damage in solid state detectors. This will enable predictive insight of the electrical behavior of detectors, aiming at their performance optimization.

\section{Modeling the radiation damage effects within the TCAD environment}

According to the premise, in order to reproduce the electrical behavior of irradiated devices, a limited set of effective, physically meaningful radiation-induced defects have been taken into account, whose parameterization allows a realistic device-level modeling and electrical behavior description in terms of space-charge, electric field profile and charge collection properties.

Radiation damage effects can be mainly classified in ionizing and non-ionizing. Ionizing effects include effects on oxide and dielectrics material, and at the silicon/oxide interface region. They are usually referred as "surface damage" and they are responsible of the build-up of trapped charge in the oxide, the increase in the number of bulk oxide traps and the increase in the number of interface traps. For TCAD simulation purposes, such effects can be accounted for in terms of fixed oxide charge $\left(Q_{O X}\right)$ and $\mathrm{SiO}_{2} / \mathrm{Si}$ interface trap states densities $\left(N_{I T}\right)$.

On the other hand, non-ionizing effects are referred as "bulk damage" and are caused by displacement of crystal atoms which therefore lead to silicon lattice defects generation, point and cluster defects formation and hence an increase of deep-level trap states. On a macroscopic scale, 
damages in solid state detectors cause an increase of leakage current (increase in noise), a changing in material resistivity, the worsening of the charge collection properties due to trapping mechanism and eventually, the decrease of the carrier's mobility and their lifetime. A deep understanding of the global radiation damage effects at both surface and bulk level has the utmost importance and the close interaction between device-level simulations and dedicated measurement campaigns on test structures may help to this purpose. In the last few decades extensive simulations and experimental studies have been carried out to understand the mechanisms of radiation damage in solid-state silicon sensors for High-Energy Physics experiments [1-6]. The unprecedented fluences to be taken into account impose new challenges for the design of effective, radiation tolerant silicon detectors and therefore the numerical modeling schemes already developed and validated at typical LHC fluences have to be reformulated.

Within the TCAD environment the most suitable physical models can be selectively activated together with the trapping mechanisms, accounting for new experimental evidences which cannot be neglected at these very high fluences, such as charge multiplication and avalanche effects.

From the TCAD stand-point, the trapping mechanisms can be described by the ShockleyRead-Hall (SRH) statistics which calculate the effect of a single defect once its type (acceptor or donor), concentration, energy distribution and capture cross sections for both electrons and holes are known. The correct trap parametrization is therefore of utmost importance in order to correctly describe the radiation damage effects. Independently of the energy distribution, acceptor traps are considered uncharged when empty while negatively charged when occupied, within the TCAD environment. They carry the charge of one electron when fully occupied. Dually, donor traps are uncharged when empty, while positively charged when occupied and they carry the charge of one hole when fully occupied. The trap occupancy function relies on the Fermi-Dirac statistics making more efficient an acceptor (donor) trap state when located below (above) the Fermi level. The trap type definition should be therefore carefully considered, especially when describing interface trap states which typically act as amphoteric traps, since they simultaneously behave as acceptor-like and donor-like traps by interacting with both free electrons and free holes [7].

\subsection{Modeling the surface radiation damage effects}

Surface damage is responsible for the surface generation current variation, strongly affecting the breakdown voltage, the inter-electrode isolation and capacitance, and it might also have a significant impact on the charge collection properties of solid-state sensors.

For general modeling purposes, surface damage effects can be mainly characterized by two parameters: oxide charge $\left(Q_{O X}\right)$ and acceptor/donor-like interface trap states $\left(N_{I T}\right)$. These parameters represent the measurable key quantities to evaluate the surface damage extent, which in turn can be translated into the radiation-induced deviation from the electrical behavior of nonirradiated devices. Their values can be assessed starting from measurements performed on dedicated test structure depending on irradiation conditions (e.g. dose and dose rate), biasing conditions during irradiation and post-irradiation processes (e.g. time/temperature of annealing). By following the procedure described in Nicollian [8] it is possible to quantitatively evaluate, thanks to $\mathrm{C}-\mathrm{V}$ measurements carried out at different frequencies on MOS structures (High-Low $\mathrm{C}-\mathrm{V}$ method), interface defects by looking at the acceptor-like trap states effects on devices manufactured on n-type substrates (p-in-n MOS capacitors, gated-diodes (GD) and nMOSFETs) 
and at donor-like trap states effects from measurements on p-type substrate devices (n-in-p MOS capacitors, GDs and pMOSFETs). The comprehensive effect of $N_{I T}$ and $Q_{O X}$ at a given dose can be e.g. assessed from the radiation-induced shifts in flatband voltage $\left(V_{F B}\right)$ on MOS capacitors for each irradiation dose. Moreover, combined effects of oxide-charge and interface traps can be in turn disentangled by means of specific measurement set-up and data analysis procedure such as the method proposed by McWhorter et al. in [9].

Eventually, these measurable dose-dependent parameters can be then fed as inputs to the TCAD simulation tools. The recently numerical modeling scheme which accounts for the surface radiation damage effects has been developed within the framework of the "new Perugia Model". It mainly relies on two uniform defect energy band distributions: the first one accounts for the acceptor-like trap states near the conduction band while the second one accounts for the donorlike defects near the valence band. Accordingly to measurements, the acceptor-like band extends from the conduction band edge to mid-bandgap, while the donor-like band is $0.6 \mathrm{eV}$ width and it extends from the valence band edge [10].

\subsubsection{Experimental procedure}

Devices under study include: (a) MOS capacitors, gate-controlled diodes (GDs) and MOSFETs fabricated at Fondazione Bruno Kessler (FBK, Italy) on 6" n-type and p-type SiSi Direct Wafer Bonded Wafers from ICEMOS Technology Ltd, Float Zone $<100>$ oriented wafer, with a nominal resistivity higher than $3 \mathrm{k} \Omega \cdot \mathrm{cm}$; (b) MOS capacitors and gated-diodes fabricated at Hamamatsu Photonics (HPK) on 6" high-resistivity p-type Float Zone, $<100>$ oriented wafer, with $\mathrm{p}$-stop or with $\mathrm{p}$-spray layer implanted at the surface to isolate adjacent $\mathrm{n}^{+}$electrodes; (c) two different processes of Infineon Technology (IFX): (i) IFX devices on high-resistivity p-type Float Zone (FZ) $\langle 100\rangle$ 8" wafers (ii) IFX devices on high-resistivity p-type Float Zone(FZ) $\langle 100\rangle 6$ " wafers with different processes and thermal budget. Further details about the test structures description and the experimental procedure and measurement setup are detailed explained in previous related works [11-13].

The validation of the "new Perugia Model" for the surface related damage effects mainly relies on the comparison between simulations and experimental data in terms of current-voltage $(\mathrm{I}-\mathrm{V})$ and capacitance-voltage $(\mathrm{C}-\mathrm{V})$ analyses of dedicated test structures exposed to X-rays irradiation with doses in the range 0.05 to $100 \mathrm{Mrad}\left(\mathrm{SiO}_{2}\right)$. The extensively reported good agreement between simulation results and measurements would bolster the use of the model as inspecting support to analyse the effect of radiation damage from the electrical behavior point of view [11,14]. Moreover, starting from the look-up table of the extrapolated measured parameters at different doses for the oxide charge $Q_{O X}$, the acceptor-like interface traps $N_{I T}{ }_{A C C}$ and the donorlike interface traps $N_{I T_{D O N}}$, it is possible to create an analytical model thus enabling predictive analyses at different doses from the investigated ones. The model can be represented by the following system of equations:

$$
\left\{\begin{array}{c}
Q_{O X}(\Phi)=Q_{O X}(0)+\Delta Q_{O X}(\Phi) \\
N_{I T_{A C C}}(\Phi)=N_{I T_{A C C}}(0)+\Delta N_{I T_{A C C}}(\Phi) \\
N_{I T_{D O N}}(\Phi)=N_{I T_{D O N}}(0)+\Delta N_{I T_{D O N}}(\Phi)
\end{array}\right.
$$

where $Q_{O X}, N_{I T_{A C C}}$ and $N_{I T_{D O N}}$ are modelled, at each irradiated dose, as the sum between the value before irradiation and the radiation-induced increase $\Delta$. The specific value of $Q_{O X}, N_{I T} A C C$ and 
$N_{I T_{D O N}}$ depends upon the technology, however the overall developed modeling scheme represented in Eq. 1 is technology-independent. Experimental evidences of the radiation-induced increase in $Q_{O X}$, and $N_{I T}$ as a function of the dose are reported in previous related works [11,22,24] for different technologies and manufacturing processes implemented by Hamamatsu Photonics (HPK, Japan) and Infineon Technologies (IFX, Austria). $Q_{O X}$ and $N_{I T}$ increase with increasing radiation levels up to about $10 \mathrm{Mrad}\left(\mathrm{SiO}_{2}\right)$ after which start to show a saturating behavior despite the dose increasing.

\subsubsection{Model sensitivity to $Q_{O X}$ and $N_{I T}$}

The worth of this model is that it accepts as input parameters the measured acceptor or donor interface trap state density $\left(N_{I T}\right)$ values depending on the substrate type. This section deals with p-type substrate but the discussion can also be extended to other typology of substrates. The sensitivity analysis reported in this paragraph aims to be qualitative and therefore valid independently of dose and technology. The $V_{F B}$ voltage can be considered as a reference point, for both MOS capacitors and gated-diodes to compare changings. Starting from a p-type substrate it is possible to analyse the sensitivity of C-V characteristics of a MOS capacitor to $N_{I T}$ in terms of low-frequency $\left(C_{L F}, f=1 \mathrm{~Hz}\right)$ and high-frequency $\left(C_{H F}, f=100 \mathrm{kHz}\right)$. By sweeping the device from inversion to accumulation $(0 \div-80 \mathrm{~V}), C_{H F}$ reflects the charge variation within the depletion layer as a function of the gate voltage. Acceptor-like interface trap states have no effects on the $\mathrm{C}-\mathrm{V}$ curves of p-type MOS capacitors, since they are traps for electrons and therefore affect the $\mathrm{C}-\mathrm{V}$ behavior limited to the strong depletion and inversion regime (Figure 1). On the other hand, Figure 2 shows how the increase/decrease of the donor-like $N_{I T}$ significantly changes the $\mathrm{C}_{\mathrm{HF}}-\mathrm{V}$ slope and thus the $V_{F B}$. Donor-like defects entrap holes, therefore the transition from depletion to accumulation is slowed down with the increasing of the donor $N_{I T}$ and always more negative values for the gate voltage are required to switch the device toward the accumulation. The $Q_{O X}$ value is chosen to reproduce the experimentally assessed $V_{F B}$ of MOS capacitors. $Q_{O X}$ represents a fixed positive charge at the $\mathrm{SiO}_{2} / \mathrm{Si}$ interface which the gate voltage has to counterbalance to switch the device form inversion to accumulation. As a consequence, variations on $Q_{O X}$ reflects into a rigid translation of the $\mathrm{C}-\mathrm{V}$ characteristics, as reported in Figure 3.

The values for $N_{I T_{D O N}}$ and $Q_{O X}$ thus established, are then straightforwardly applied to the simulation of different test structures, e.g. the gated diodes. The I-V characteristics of gated diodes is sensitive to both the interface trap type acceptor and donor. Therefore the $N_{I T_{A C C}}$ value is chosen to reproduce the measured curve at a specific dose. Figure 4 shows the sensitivity of the gated diode I-V curve to $N_{I T_{A C C}}$ (blue curves) and $N_{I T_{D O N}}$ (red curves) variations from the reference values (black curve). Solid lines refer to an increase of $N_{I T}$ while dashed lines refer to a decrease of the same quantity within a $\pm 20 \%$ from the reference values. An increasing in $N_{I T_{A C C}}$ and $N_{I T_{D O N}}$ reflects into a combined stretch and shift of the overall I-V curve towards the inversion and accumulation operating regions, respectively. On the contrary, a decrease in $N_{I T}$ ACC and $N_{I T_{D O N}}$ limits the I-V curves amplitude. The I-V curve amplitude is directly correlated to $N_{I T}$ by the surface velocity [15], which represents a key parameter to validate the TCAD modeling scheme.

The described methodology has been pursued for the development of the "new Perugia Model" accounting for the surface radiation damage effects. It relies on physically meaningful parameters assessed from measurements (i.e. $N_{I T}$ and $Q_{O X}$ ) to reproduce the measured curves. 
The proposed modeling scheme has been extensively validated against measurements and can be applied for the analysis and prediction of the radiation damage effects of different classes of devices $[10,11,22,24]$.

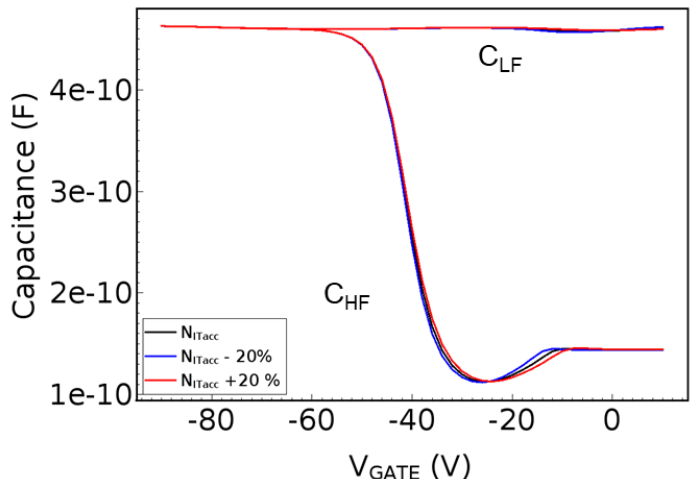

Figure 1: MOS capacitor: sensitivity to the variation of acceptor $N_{I T}$ ACC only, within a $\pm 20 \%$ from the reference value (black).

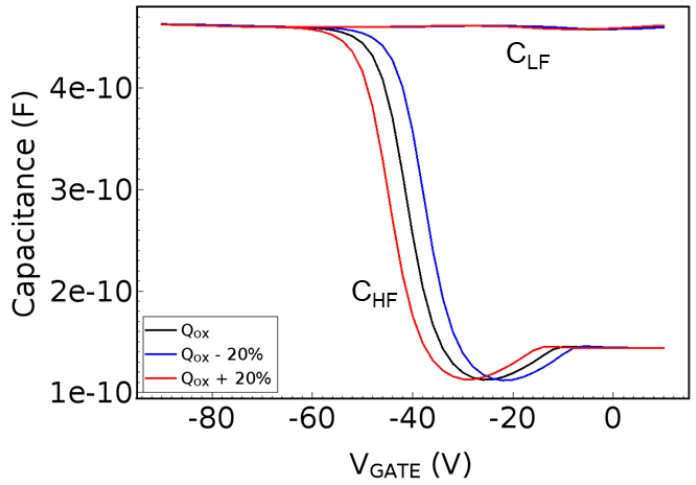

Figure 3: MOS capacitor: sensitivity to the variation of $Q_{O X}$ only, within a $\pm 20 \%$ from the reference value (black).

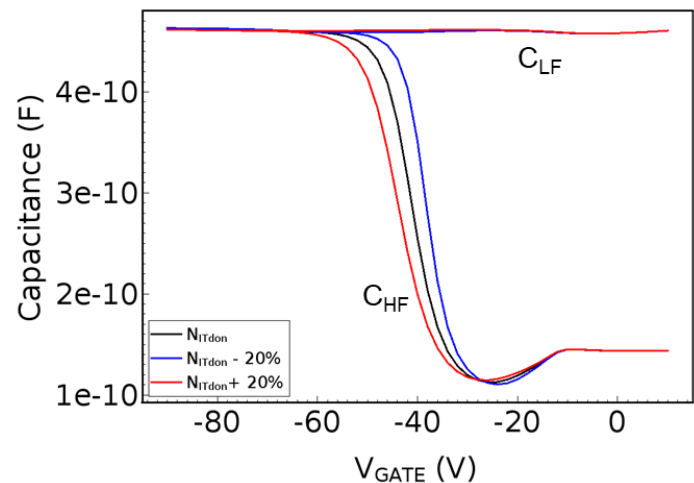

Figure 2: MOS capacitor: sensitivity to the variation of donor $N_{I T_{D O N}}$ only, within a $\pm 20 \%$ from the reference value (black).

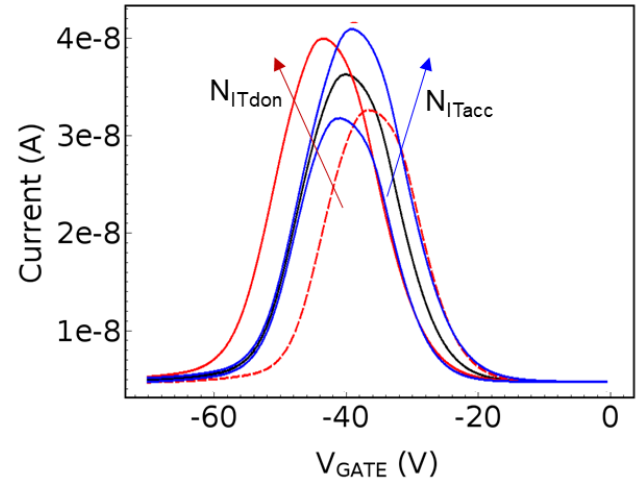

Figure 4: Gated diode: sensitivity to $N_{I T} A C C$ (blue) and $N_{I T_{D O N}}$ (red) variations. The arrows indicate the increasing of the $N_{I T}$ within a $\pm 20 \%$ from the reference values (black).

\subsection{Bulk damage modeling}

The radiation-induced electrically active defects alter the effective space charge of a sensor, thus leading to variations in the electric field distribution within the device and to the increase of the depletion voltage. Typically, silicon-based particle detectors are designed to operate in full depletion regime and the radiation damage effects force higher operation voltages in order to avoid under-depletion and loss of active volume, and therefore signal. Defects can contribute with positive (donors) or negative (acceptors) charge to the effective space charge, altering the electric field distribution and the depletion voltage of a device. Inhomogeneous distribution of space charge might lead to an electric field with two main peaks at front and back contact. The peaks properties highly depend on the particle type used for the irradiation experiment (e.g. neutron and/or proton damage) [16].

The RD50 collaboration widely investigated the radiation damage effects of irradiated devices and Moll [17] gives and overview of the most relevant defects within the silicon bandgap. 
However, within the TCAD environment it is not possible to include the whole set of measured defects because this methodology leads to numerical convergence related problems. The methodology proposed in this works aims at reproducing the sensor space-charge by means of the introduction, within the silicon bandgap, of a limited number of acceptor and donor trap states whose net effect is compatible with the measured one. To this end, macroscopic quantities such as I-V characteristics and charge collection properties have been considered as figures of merit.

\subsubsection{Two vs. three levels modeling}

For charge collection properties investigation, two simulated layouts have been considered, e.g. a n-on-p strip $300 \mu \mathrm{m}$ depth and a PiN diode $50 \mu \mathrm{m}$ depth. The strips are AC-coupled sensors with a $80 \mu \mathrm{m}$ strip pitch. Signals in the sensors are induced by fast electrons from a ${ }^{90} \mathrm{Sr}$ source. The signal in the PiN diodes have been instead induced by a laser $1064 \mathrm{~nm}$ at room temperature. Further information about the strips and PiN diodes design and measurement setup are are described in detail in [18] and [19], respectively. Both n-on-p strip detectors and PiN diodes have been simulated by means of a $2 \mathrm{D}$ layout, with a substrate doping concentration of $3.3 \times 10^{12} \mathrm{~cm}^{-3}$ (p-type).

First of all, we considered only two significant bulk trap levels in addition to the already mentioned traps for the surface damage effects. In particular, an acceptor trap level at $0.42 \mathrm{eV}$ from the conduction band and a donor trap level at $0.23 \mathrm{eV}$ from the conduction band have been considered [17]. The concentration of the trap states closely depends on the irradiation fluence by the so-called introduction rate $\left(\eta-\mathrm{cm}^{-1}\right)$. By slightly varying this parameter a significant effect in terms of device electrical behavior has been pointed out, e.g. in terms of electric field distribution. On the basis of experimental evidences [16], the electric field distribution is properly described with a "double peak" behavior shape. As inferred from experimental measurement, the "balance" between the two electric field peaks closely depends on the particle type used for the irradiation. Sensors irradiated with neutrons show a dominant peak on the junction side. This can be modelled, within the TCAD environment, by considering an excess of acceptor trap states respect to the donor. To begin with, an introduction rate of $0.2 \mathrm{~cm}^{-1}$ and $0.9 \mathrm{~cm}^{-1}$ for the acceptor and donor trap states have been considered respectively and the obtained reference curves are shown in red in Figure 5 and 6 . The decreasing of the acceptor introduction rate $\left(\eta_{A C C}\right)$ only, reflects in a less pronounced electric field peak on the junction side, while the ohmic side peak starts to increase. In general, the acceptor and donor trap states tend to compensate each other, and the donor becomes dominant by decreasing the acceptor concentration (i.e. $\eta_{A C C}$ ). On the other hand, the overall effect obtained by decreasing $\eta_{D O N}$ is a predominant electric field peak on the junction side (Figure 6). With only two trap levels it is therefore possible to obtain a realistic electric field distribution composed by the two peaks at the junction side and at the ohmic contact side [23]. This modeling scheme, however, can fail when trying to reproduce the increase in the leakage current with the fluence and the charge collection properties. Since the leakage current is mostly produced by defect levels close to the middle of the bandgap, an additional third level has been included (Acceptor level at $\mathrm{E}_{\mathrm{C}}-0.46 \mathrm{eV}$ [17]). This level adds a homogeneous additional negative space charge that does not affect the two-peaks electric field distribution. The simulation results of the updated modeling scheme for the bulk damage are in good agreement with measurements in terms of charge collection efficiency and value of the damage constant $(\alpha)$. The leakage current 
$(\Delta I)$ over a detector volume $(V)$ is proportional to the irradiated fluence $\left(\Phi_{\text {eff }}\right)$ with a proportionality factor called damage constant $(\alpha)$, as described in eq. 2 .

$$
\alpha \cdot \Phi_{\text {eff }}=\frac{\Delta I}{V}
$$

Measurements report values for $\alpha \sim 4 \div 7 \times 10^{-17} \mathrm{~A} / \mathrm{cm}^{3}$ depending on the annealing time/temperature [21]. Since the damage constant simulated at $+20^{\circ} \mathrm{C}$ is $3.5 \times 10^{-15} \mathrm{~A} / \mathrm{cm}^{3}$ we have simulated $\alpha$ at $-20^{\circ} \mathrm{C}$ and then scaled to $+20^{\circ} \mathrm{C}$ by means of the Chilingarov formula [20], consistently with the typical measurements procedure. By following this process, the simulated $\alpha$ $=5.4 \times 10^{-17} \mathrm{~A} / \mathrm{cm}^{3}$ for the simulated three-levels bulk damage modeling scheme. Since the required bias voltage to fully deplete the device would become unrealistically high, we limited the reported $\alpha$ values to a fluence of $3.0 \times 10^{15} \mathrm{n}_{\mathrm{eq}} / \mathrm{cm}^{2}$.

Eventually, experimental data and simulation findings have been compared aiming at the modeling scheme validation. The charge collection as a function of the fluence has been simulated for strips and PiN diodes structures and then compared with experimental data carried out by Affolder [18] and RD50 collaboration [19] respectively. The Affolder's experimental data are collected charge measurements of planar silicon detectors biased at $900 \mathrm{~V}$ after irradiation with neutrons and $26 \mathrm{MeV}$ protons up to $2.2 \times 10^{16} \mathrm{n}_{\mathrm{eq}} / \mathrm{cm}^{2}$. Instead, the Ferrero's measurements have been performed with three different intensities of a $1064 \mathrm{~nm}$ pulsed laser and then normalized to the non-irradiated collected charge in order to obtain the charge collection efficiency. The good agreement between simulation and measurements for all the considered irradiation fluences, qualifies the validity of the method and the comprehensiveness of the selected modeling scheme for provisional analysis of irradiated silicon detectors, at the same time assessing the "nonuniqueness" of a comprehensive combined surface and bulk TCAD radiation damage modeling scheme. Table 1 and 2 summarize the comprehensive bulk and surface TCAD numerical modelling scheme.

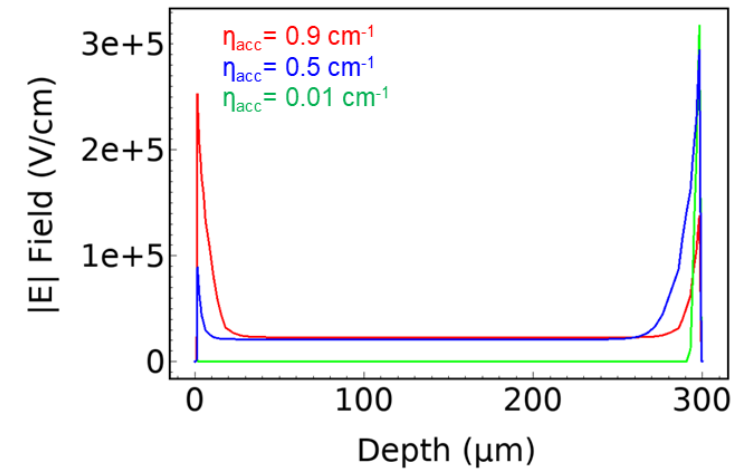

Figure 5: Strip structure: longitudinal electric field distribution across the device biased at $-900 \mathrm{~V}$ and irradiated at $2.0 \times 10^{16} \mathrm{n}_{\mathrm{eq}} / \mathrm{cm}^{2}$. Different values of the acceptor trap state introduction rate $\left(\eta_{A C C}\right)$ have been considered, while $\eta_{D O N}$ is $0.2 \mathrm{~cm}^{-1}$.

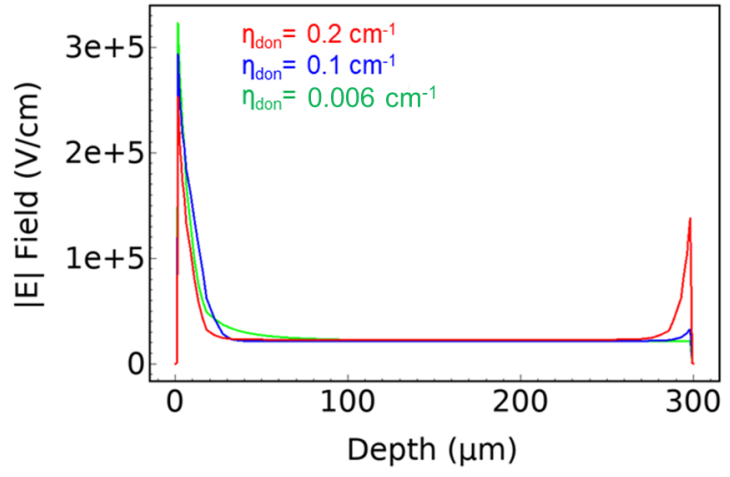

Figure 6: Strip structure: longitudinal electric field distribution across the device biased at $-900 \mathrm{~V}$ and irradiated at $2.0 \times 10^{16} \mathrm{n}_{\mathrm{eq}} / \mathrm{cm}^{2}$. Different values of the donor trap state introduction rate $\left(\eta_{D O N}\right)$ have been considered, while $\eta_{A C C}$ is $0.9 \mathrm{~cm}^{-1}$. 


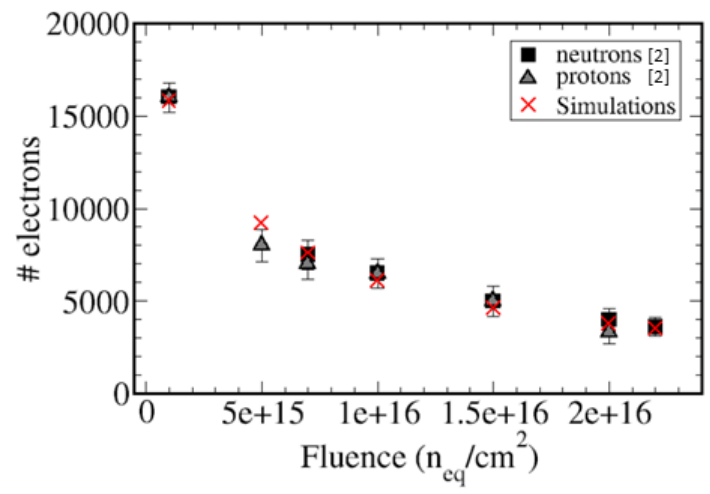

Figure 8: Charge collection as a function of fluence: simulations (three-levels model) vs. measurements at different fluences for strip detectors (measurements data from [18]).

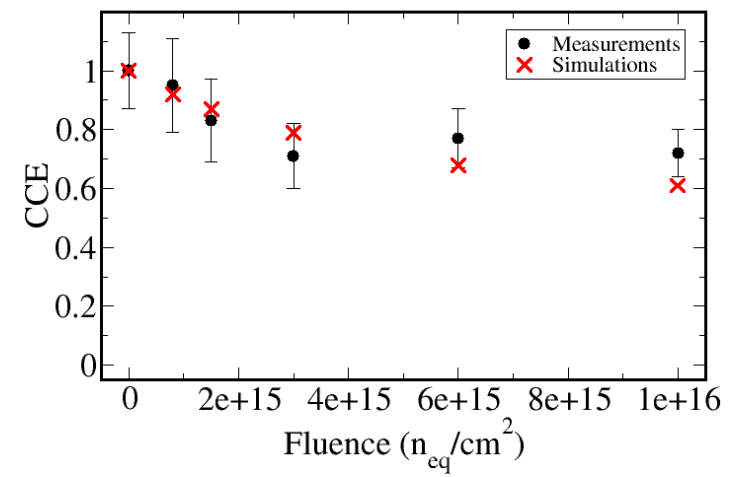

Figure 9: Charge collection as a function of fluence: simulations (three-levels model) vs. measurements at different fluences for PiN diodes devices (measurements data from [19]).

Table 1: The bulk radiation damage model. The energy, the introduction rate $(\eta)$ and the cross-sections for electrons $\left(\sigma_{\mathrm{e}}\right)$ and holes $\left(\sigma_{\mathrm{h}}\right)$ are reported for all the three trap state levels.

\begin{tabular}{|l|c|c|c|c|}
\hline Trap Type & Energy $(\mathbf{e V})$ & $\boldsymbol{\eta}\left(\mathbf{c m}^{-1}\right)$ & $\boldsymbol{\sigma}_{\mathbf{e}}\left(\mathbf{c m}^{\mathbf{2}}\right)$ & $\boldsymbol{\sigma}_{\mathbf{h}}\left(\mathbf{c m}^{\mathbf{2}}\right)$ \\
\hline Donor & $\mathrm{E}_{\mathrm{C}}-0.23$ & 0.006 & $2.3 \times 10^{-14}$ & $2.3 \times 10^{-15}$ \\
\hline Acceptor & $\mathrm{E}_{\mathrm{C}-}-0.42$ & 1.613 & $1.0 \times 10^{-15}$ & $1.0 \times 10^{-14}$ \\
\hline Acceptor & $\mathrm{E}_{\mathrm{C}-}-0.46$ & 0.900 & $7.0 \times 10^{-15}$ & $7.0 \times 10^{-14}$ \\
\hline
\end{tabular}

Table 2: The surface radiation damage model. The acceptor and donor trap states are uniform bands whose energy range is determined by $\mathrm{E}_{T} . \mathrm{E}_{C}$ and $\mathrm{E}_{V}$ are the conduction and valence band edges, respectively. The reported concentration values refer to saturation, namely doses higher than $10 \mathrm{Mrad}(\mathrm{SiO} 2)$.

\begin{tabular}{|l|c|c|c|c|}
\hline Trap Type & Energy $(\mathbf{e V})$ & Concentration $\left.\mathbf{( c m}^{-2}\right)$ & $\boldsymbol{\sigma e}_{\mathbf{e}}\left(\mathbf{c m}^{\mathbf{2}}\right)$ & $\boldsymbol{\sigma}_{\mathbf{h}} \mathbf{( \mathbf { c m } ^ { 2 } )}$ \\
\hline Fixed Charge & - & $1.08 \times 10^{12}$ & - & - \\
\hline Donor & $\mathrm{E}_{\mathrm{V}}<\mathrm{E}_{\mathrm{T}}<\mathrm{E}_{\mathrm{V}}+0.6$ & $1.20 \times 10^{12}$ & $1.0 \times 10^{-15}$ & $1.0 \times 10^{-16}$ \\
\hline Acceptor & $\mathrm{E}_{\mathrm{C}}-0.56<\mathrm{E}_{\mathrm{T}}<\mathrm{E}_{\mathrm{C}}$ & $1.35 \times 10^{12}$ & $1.0 \times 10^{-16}$ & $1.0 \times 10^{-15}$ \\
\hline
\end{tabular}

\section{Conclusion}

This work focused on the methodology for the development of a numerical model, fully implemented within the TCAD environment, aiming at describing the comprehensive bulk and surface damage effects induces by radiation in silicon sensors conceived as particle detectors. Once validated, the model can be proficiently applied for predictive insight of the detectors electrical $t$ only different design options/detector geometries can be evaluated, but also different vendors and/or technology options can be investigated. The predictive capabilities of the new University of Perugia TCAD model have been eventually applied to HL-LHC radiation damage framework, fostering its application to the analysis and optimization of different classes of detectors to be used in the future HEP experiments.

\section{References}

[1] A. Morozzi et al., A combined surface and bulk TCAD damage model for the analysis of radiation detectors operating at HL-LHC fluences, JINST 11 C12028, 2016.

[2] F. Moscatelli et al., Measurements and TCAD simulations of bulk and surface radiation damage effects in silicon detectors, IEEE Trans. Nucl. Sci., vol. 63, no. 5, 2016, pp. 2716-2723. 
[3] G. Jain, Radiation Damage Modelling: TCAD Simulation, PoS(Vertex 2018) 017.

[4] J. Schwandt et al., A new model for the TCAD simulation of the silicon damage by high fluence proton irradiation, arXiv:1904.10234 [physics.ins-det].

[5] R. Dalal et al., Combined effect of bulk and surface damage on strip insulation properties of proton irradiated n+-p silicon strip sensors, JINST., vol. 9, number P04007, 2014.

[6] D. Pennicard, G. Pellegrini, C. Fleta, R. Bates, V. O’Shea, C. Parkes, N. Tartoni, Simulations of radiation-damaged $3 D$ detectors for the SuperLHC, NIMA, vol. 592, pp 16-25, 2008.

[7] S.T. Chang, J.K. Wu, and S.A. Lyon, Amphoteric defects at the Si-SiO2 interface, Appl. Phys. Lett., vol. 48 , no. 662,1986 , pp. 662-664.

[8] E.H. Nicollian and J. R. Brews, MOS (Metal Oxide Semiconductor), Physics and technology, John Wiley and Sons, 1982, pp. 319-356.

[9] P.J. McWhorter and P.S. Winokur, Simple technique for separating the effects of interface traps and trapped-oxide-charge in metal-oxide semiconductor transistors, Appl. Phys. Lett., vol. 48, 1986, pp. 133-135.

[10] D Passeri et al., Modeling radiation damage in TCAD, PoS(Vertex 2017) 046.

[11] F. Moscatelli et al., Measurements and simulations of surface radiation damage effects on IFX and HPK test structures, https://doi.org/10.1016/j.nima.2019.162794.

[12] F. Moscatelli et al, Surface damage characterization of FBK devices for High Luminosity LHC (HL-LHC) operations, JINST 12 P12010, 2017.

[13] F. Moscatelli et al., Effects of Interface Donor Trap States on Isolation Properties of Detectors Operating at High-Luminosity LHC, IEEE Trans. Nucl. Sci., vol. 64, no. 8, 2017, pp. 2259-2267.

[14] D. Passeri et al., Modeling of radiation damage effects in silicon detectors at high fluences HL-LHC with Sentaurus TCAD, NIMA Vol. 824 (2016), pp. 443-445.

[15] G. Verzellesi et al., On the accuracy of generation lifetime measurement in high-resistivity silicon using PN gated diodes, IEEE Trans. Electron Devices, vol. 46, no. 4, 1999, pp. 817-820.

[16] G. Kramberger, Reasons for high charge collection efficiency of silicon detectors at HL-LHC fluences, NIMA 924 (2019), pp. 192-197.

[17] M. Moll, Displacement Damage in Silicon Detectors for High Energy Physics, IEEE Trans. Nucl. Sci., vol. 65 , no. 8, 2018.

[18] A. Affolder et al, Collected charge of planar silicon detectors after pion and proton irradiations up to $2.2 \times 10^{16}$ neqcm $^{-2}$, NIMA Vol. 623 (2010), pp. 177-179.

[19] M. Ferrero, 34th RD50 Workshop, June 12-14 2019.

[20] A. Chilingarov, Generation current temperature scaling, RD50 technical note.

[21] A. Dierlamm, KIT Status, CMS Outer tracker Meeting, March 2019.

[22] F. Moscatelli, Analysis of surface radiation damage effects at HL-LHC fluences: Comparison of different technology options, NIMA Vol. 924 (2019), pp. 198-202.

[23] V. Eremin et al., The origin of double peak electric field distribution in heavilyirradiated silicon detectors, NIMA 476 (2002), pp. 556-564.

[24] A. Morozzi et al., Characterization of irradiated p-type silicon detectors for TCAD surface radiation damage model validation, 2020 JINST 15 C01029. 